\title{
Secondary Patellar Resurfacing after Primary Bicondylar Knee Arthroplasty did Not Meet Patients' Expectations
}

\author{
João Correia, Marc Sieder, Daniel Kendoff, Mustafa Citak, Thorsten Gehrke, Wolfgang Klauser and \\ Carl Haasper
}

Helios ENDO-Klinik Hamburg, Hamburg, Germany

\begin{abstract}
Secondary patella resurfacing is a controversial procedure which is applied in patients with anterior knee pain after a bicondylar knee arthroplasty (with unresurfaced patella). A group of 46 patients were submitted to this procedure and their satisfaction, range of motion and pain improvement was evaluated. $52.2 \%$ of the patients were satisfied with the procedure, with an improvement in pain (Visual Analogue Scale) of $65 \%$ and an improvement in range of motion in $56,5 \%$, with roundabout half of the patients having no resolution to their complaints. Whilst an improvement was not achieved in all patients, as it was initially hypothesised, this procedure should be considered when a revision knee arthroplasty is performed with an unresurfaced patella.
\end{abstract}

Keywords: Anterior knee pain, bicondylar arthroplasty, revision knee arthroplasty, secondary patella resurfacing, unresurfaced patella.

\section{INTRODUCTION}

The first record of resurfacing of the patella performed in combination with a condylar resurfacing arthroplasty was performed by Groeneveld in 1970 [1, 2], with most knee replacement systems having an option for patella resurfacing since the beginning of the 1980s [2, 3].

In the literature, opinions vary when comparing the surgical practice applied, with the Swedish registry revealing resurfacing in $14 \%$ of the cases [4], whilst in the USA and Denmark, resurfacing is applied in $76 \%$ of the cases $[4,5]$.

In revision surgery of total knee prosthesis, secondary resurfacing of the patella is controversial as well. Usually performed in patients with anterior knee pain, where the patella was unresurfaced in the index procedure, improvements are expected in less than $60 \%$ of patients [3, 6-8]. The reasons for this are unproven for now, with a number of studies presenting conflicting information [9-12].

Many conditions surrounding the patellofemoral joint can give rise to anterior knee pain - patella maltracking, bony alterations (loose body impingement, osteophytes) and soft tissue conditions (complex regional pain syndrome, bursitis, peripatellar tendinopathy)[2] - thus making it difficult to treat.

An eventual screening tool could be the use of bone scans, with $95 \%$ of patients with anterior knee pain showing significant increased uptake ("hot patella"). The finding of a "hot patella" suggests that the problem is related to the patellofemoral joint [13]. Albeit, according to a recent study, there seems to be no relation between "hot patella" and

\footnotetext{
*Address correspondence to this author at the Helios ENDO-Klinik Hamburg Orthopaedic Surgery Holstenstraße 2, 22767 Hamburg, Germany; Tel: +49 40 3197-0; Fax: +49 40 3197-1938;

E-mail: Carl.Haasper@damp.de
}

subsequent improvement after secondary patella resurfacing $[14,15]$.

The aim of this study was to evaluate a group of patients, with bicondylar knee replacements, who were later submitted to secondary patella resurfacing, with the hypothesis that the procedure would be the resolution to their persistent anterior knee pain.

\section{MATERIAL AND METHODS}

During the period of November 2005 to October 2007, 48 patients with bicondylar knee replacements were submitted to secondary patella resurfacing. The follow-up ranged from 14 to 38 months, with 2 patients lost to followup.

The main indication for the procedure was persistent anterior pain knee (36 patients), with a secondary indication being range of motion restrictions due to arthrofibrosis (13 patients).

In total there were 18 male patients and 28 female patients, grouped according to their initial implant (bicondylar implants; rotating hinge implants; hinged implants) and further into 2 groups (patients with anterior knee pain and with arthrofibrosis).

The patients were telephonically interviewed, and enquired as to their:

- level of satisfaction - simple inquiry as to the satisfaction of the patient after the secondary resurfacing;

- $\quad$ range of motion - data recorded pre- and postoperatively and compared to what patients specified; inquiry as to the need of assistance when climbing up/down stairs;

- $\quad$ pain - Visual Analogue Scale recorded pre- and postoperatively, compared to the patients information; 
- $\quad$ pain control medication - comparison of the patient medication preoperatively and the medication referred to during the interview.

\section{Statistical Analysis}

All variables were expressed in terms of mean \pm standard deviation (SD) of the mean. A Student's $t$ test was performed when the data had a normally Gaussian distribution; otherwise the Mann-Whitney test was employed. The Shapiro-Wilk normality test was performed to ascertain whether the data were normally distributed. For all tests, $p<$ 0.05 was considered statistically significant. Statistical analysis was carried out by means of a statistical software package (GraphPad Prism Version 4.1, GraphPad Software Inc., La Jolla, CA).

\section{RESULTS}

\section{Patients}

The mean age was 65 years $(45 y-81 y)$. The mean time since the last procedure of the affected knee was between 12 months in the bicondylar implants and 18 months in the rotating hinge implants, with previous surgeries averaging 1.56 and 2.33 for the former and latter respectively (Figs. 1, 2).

The average body mass index (BMI) was 28.1 (primary implants) and 26.9 (rotating hinge implants).

\section{Patient Satisfaction}

Overall, $52.2 \%$ of the patients were satisfied with the secondary procedure (Fig. 3). When stratifying to specific implants and indications, the results were as shown (Table 1).

When correlating for age, half of the patients below the age of 70 benefitted from the procedure, while those above this age revealed no improvements.

\section{Range of Motion}

The postoperative range of motion was improved in 26 cases (Fig. 4) with no statistical significance, with varying results according to initial indications (Table $\mathbf{1}$ ).

\section{Pain and Pain Management}

The pain (VAS) improved significantly in 30 cases (Fig. 5) in both the anterior knee pain and arthrofibrosis groups, with a decrease in postoperative painkiller use in 12 cases (6 increased, 28 unchanged).

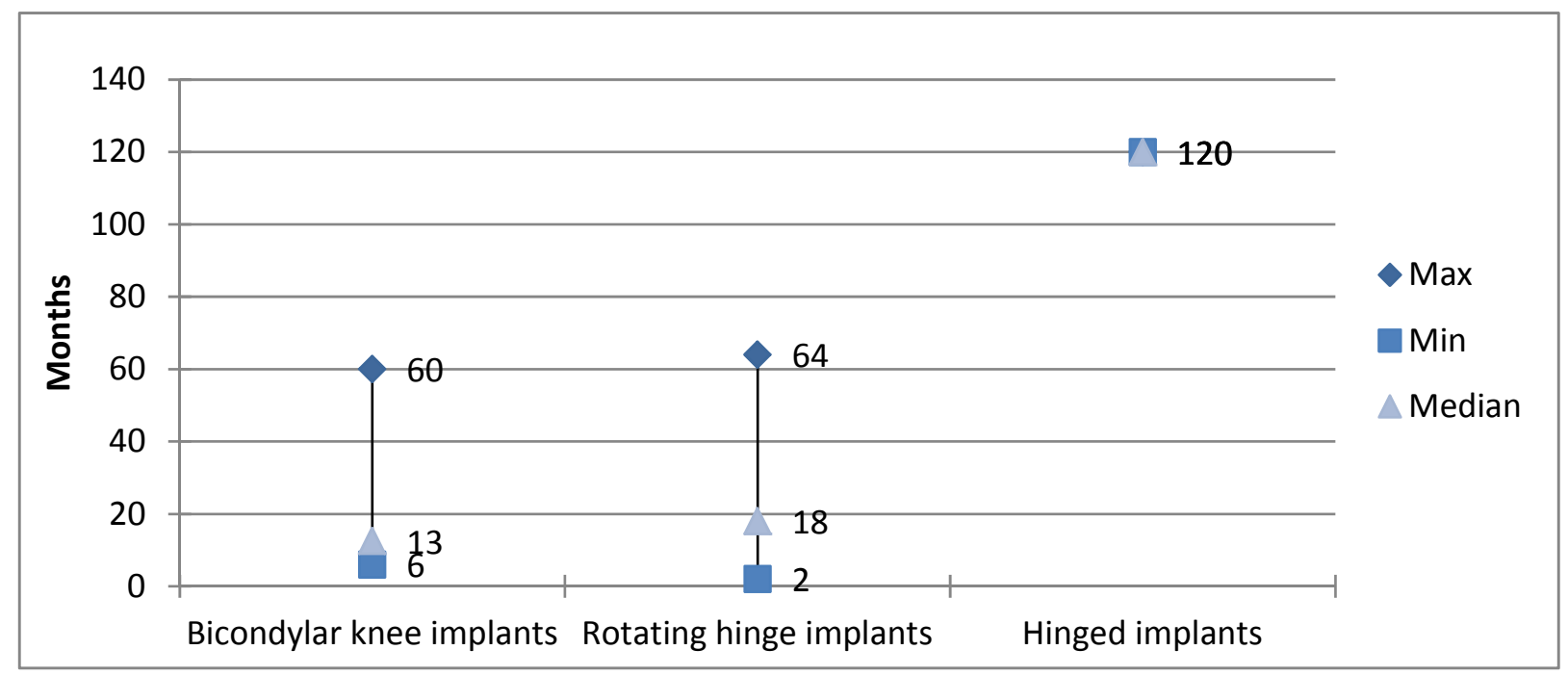

Fig. (1). Time passed since last surgery.

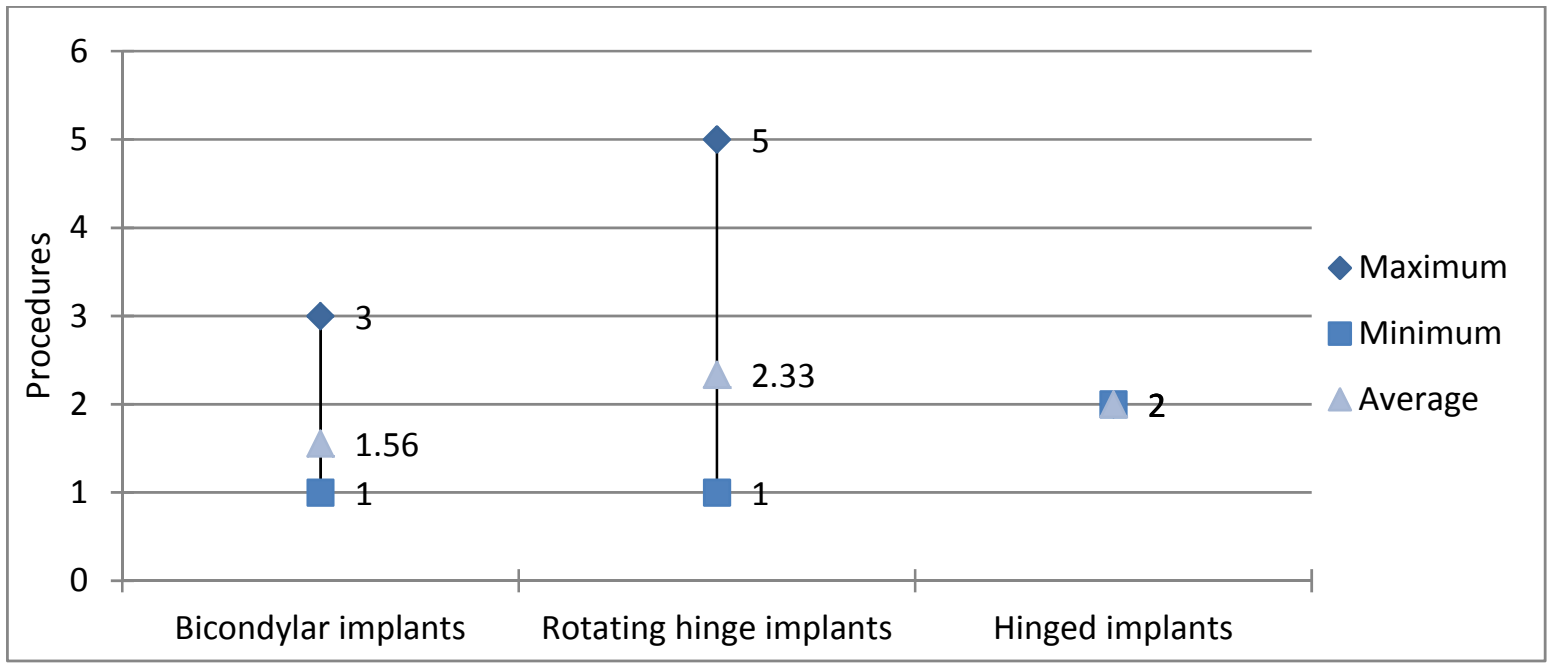

Fig. (2). Previous surgeries. 


\section{Satisfied Dissatisfied}

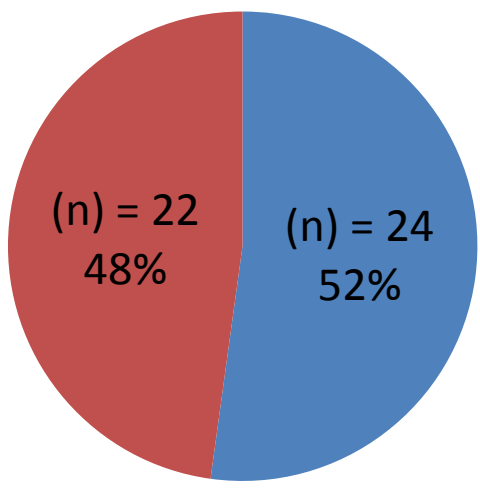

Fig. (3). General patient satisfaction.

\section{Other Procedures}

During the secondary resurfacing, patients were submitted to soft-tissue procedures (Table 2).

Four patients were submitted to further procedures during the study period, as the secondary resurfacing had led to no improvement in symptoms (2 revision arthroplasties; 1 passive knee mobilization under anesthesia; 1 arthroscopic arthrolysis).

\section{Complications}

There were 2 surgery-related complications: a wound healing complication, with the need for debridement on 3 separate occasions (due to early removal of sutures) and a periprosthetic fracture of the patella, after a fall.

\section{DISCUSSION}

Over half of the patients submitted to secondary resurfacing of the patella benefitted from the procedure, with $65.2 \%$ revealing an improvement in pain symptoms. Although our hypothesis was rejected, this procedure may be an adjuvant solution to anterior knee pain and should be considered in the preoperative planning of revision surgery.

The results reflect current literature, which reports improvements in close to $50 \%$ of cases, with secondary resurfacing of the patella [3, 6-8], but our knowledge of indicators that would reliably identify those patients who may develop AKP and how best to treat them to avoid dissatisfaction remains deficient [2]. A recent study revealed improvements in knee scores in a similar percentage of cases, with a specific cause for anterior knee pain being unknown [16].

When considering subgroups, the under-70 year old patients with anterior knee pain after bicondylar knee replacement responded well to this form of treatment. Postoperative improvement in range of motion $(60 \%)$ and improvement in pain (80\%) led to patient satisfaction of $75 \%$ in this subgroup, providing a justified indication for this

Table 1. Stratified Patient Satisfaction and Post-Operative Results

\begin{tabular}{|c|c|c|c|c|c|c|c|c|c|c|c|c|c|c|c|c|c|c|c|c|c|c|c|c|}
\hline \multirow[b]{2}{*}{ Indication } & \multicolumn{6}{|c|}{ Bicondylar Implants } & \multicolumn{6}{|c|}{ Rotating Hinge Implants } & \multicolumn{6}{|c|}{ Hinged Implants } & \multicolumn{6}{|c|}{ Total } \\
\hline & \multicolumn{3}{|c|}{ AKP } & \multicolumn{3}{|c|}{$\mathrm{AF}$} & \multicolumn{3}{|c|}{ AKP } & \multicolumn{3}{|c|}{$\mathrm{AF}$} & \multicolumn{3}{|c|}{ AKP } & \multicolumn{3}{|c|}{$\mathrm{AF}$} & \multicolumn{3}{|c|}{ AKP } & \multicolumn{3}{|c|}{$\mathrm{AF}$} \\
\hline Patient satisfaction & 10 & - & 5 & - & - & 3 & 8 & - & 9 & 5 & - & 5 & 1 & - & - & - & - & - & 19 & - & 13 & 5 & - & 8 \\
\hline PO VAS & 12 & 1 & 2 & 1 & 1 & 1 & 9 & 2 & 6 & 7 & 1 & 2 & 1 & - & - & - & - & - & $22 *$ & 3 & 8 & $8^{*}$ & 2 & 3 \\
\hline
\end{tabular}

$\mathrm{PO}$ - post-operative; $\mathrm{AKP}$ - anterior knee pain; $\mathrm{AF}$ - arthrofibrosis; I - improved; $\mathrm{W}$ - worsened; $\mathrm{U}$ - unchanged

$* \mathrm{p}<0,05 * * \mathrm{p}>0,05$.

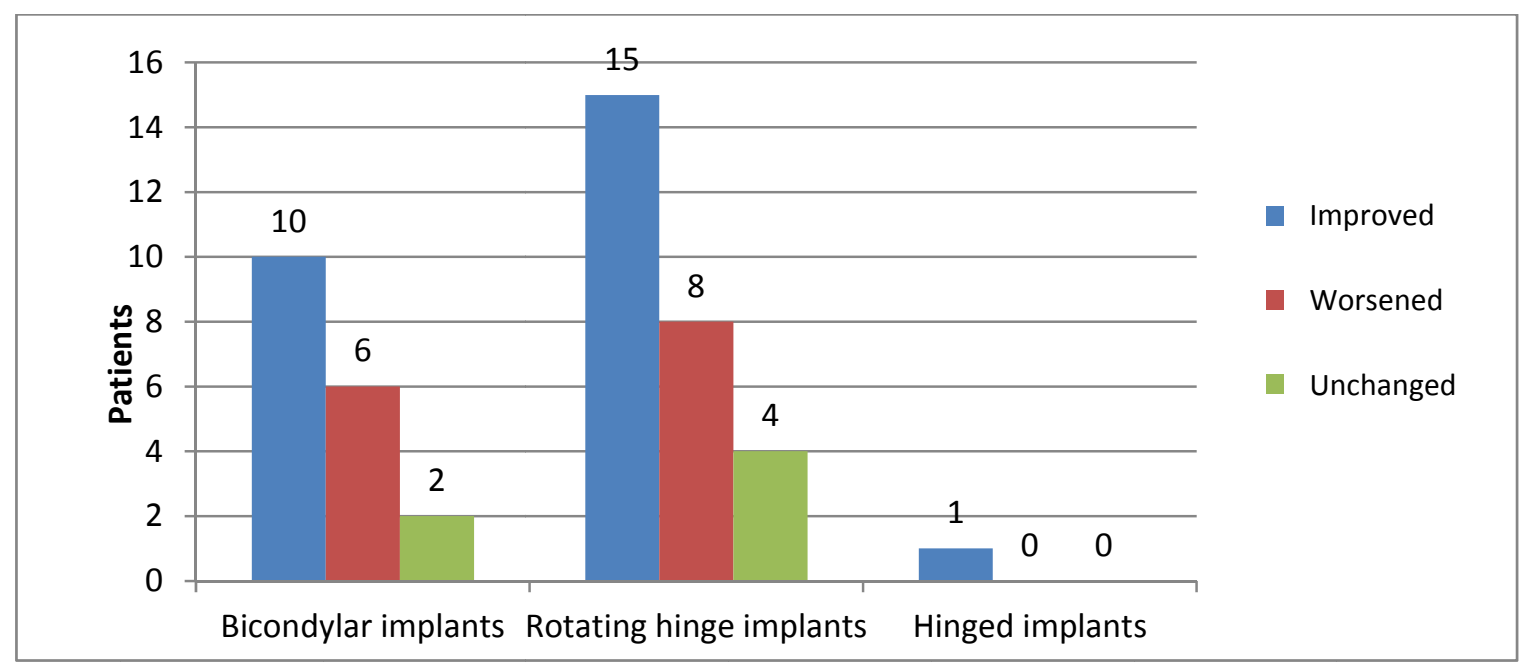

Fig. (4). Postoperative range of motion. 
Arthrofibrosis

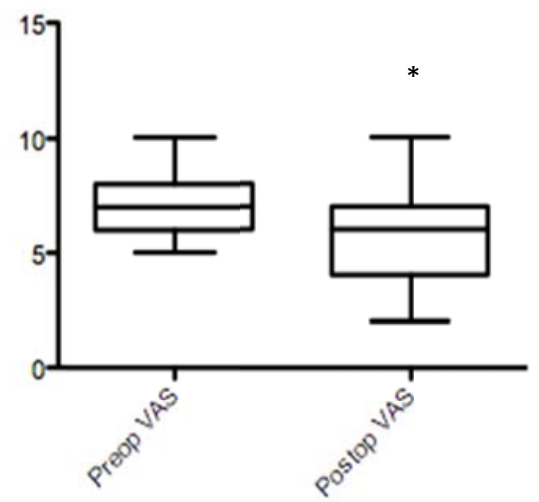

$*-p<0,05$
Anterior knee pain

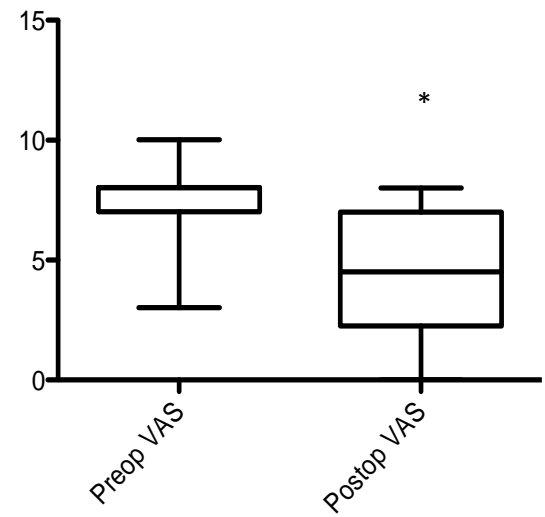

Fig. (5). - Postoperative pain evaluation (VAS).

Table 2. Soft-Tissue Procedures

\begin{tabular}{|c|c|c|c|c|c|c|c|c|}
\hline \multirow[b]{2}{*}{ Indication } & \multicolumn{2}{|c|}{ Bicondylar Implants } & \multicolumn{2}{|c|}{ Rotating Hinge Implants } & \multicolumn{2}{|c|}{ Hinged Implants } & \multicolumn{2}{|c|}{ Total } \\
\hline & AKP & $\mathrm{AF}$ & $\mathrm{AKP}$ & $\mathrm{AF}$ & AKP & $\mathrm{AF}$ & AKP & $\mathrm{AF}$ \\
\hline Lateral release & 10 & 3 & 24 & 7 & 1 & 0 & 35 & 10 \\
\hline Medial imbrication & 5 & 2 & 13 & 10 & 1 & 0 & 19 & 12 \\
\hline
\end{tabular}

$\mathrm{AKP}$ - anterior knee pain; $\mathrm{AF}$ - arthrofibrosis.

procedure.

Similarly, this indication may be applied to patients with arthrofibrosis, as a 53,8\% improvement in range of motion and $62 \%$ improvement in pain symptoms was achieved in these patients. When reviewing current literature, a number of other procedures are mentioned, such as manipulation under anesthesia and aggressive physiotherapy, arthroscopic and open arthrolysis and revision arthroplasty [17, 18], with no apparent mention of secondary patella resurfacing.

In this study, secondary resurfacing of the patella revealed a low complication rate $(2.2 \%)$ and a low rate of further procedures (10.9\%).

Whether these patients improved from the secondary resurfacing itself, or from the associated soft-tissue procedures or both, remains to be seen, with further studies necessary to differentiate between the various techniques.

A sine qua non condition for the success of a secondary resurfacing is an accurate and well-fixed primary prosthesis, with correct ligament balance.

\section{CONCLUSION}

Although secondary resurfacing did not resolve the anterior knee pain in all patients, it is definitely an important procedure to keep in mind when revising a bicondylar arthroplasty. Further studies need to be done in order to provide improved screening for the origin of anterior knee pain and to evaluate the soft-tissue procedures in these cases.

\section{ACKNOWLEDGEMENT}

No benefits in any form have been received or will be received from a commercial party related directly or indirectly to the subject of this article. No funds were received in support of this study.

\section{CONFLICT OF INTEREST}

The authors confirm that this article content has no conflicts of interest.

\section{REFERENCES}

[1] Groeneveld HB, Schollner D, Bantjes A, et al. Total knee-joint endoprosthesis saving the crucial and collateral ligaments. Z Orthop Ihre Grenzgeb 1971; 109(14): 599-607.

[2] Schindler OS. Patellar resurfacing in total knee arthroplasty. In: Scott WN, Ed. Surgery of the knee. Philadelphia: Churchill Livingstone/Elsevier 2012; pp. 1161-90.

[3] Laskin RS. The spectrum of total knee replacement. In: Laskin RS Denham RA, Apley AG, Eds. Replacement of the knee. Berlin: Springer-Verlag 1984; p. 222.

[4] Robertsson O, Bizjajeva S, Finstad A, et al. Knee arthroplasty in Denmark, Norway and Sweden. Acta Orthop 2010; 81(1): 82-9.

[5] Johnson TC, Tatman PJ, Mehle S, et al. Revision surgery for patellofemoral problems: should we always resurface?. Clin Orthop Relat Res 2012; 470(1): 211-9.

[6] Campbell DG, Mintz AD, Stevenson TM. Early patellofemoral revision following total knee arthroplasty. J Arthroplasty 1995; 10(3): 287-91.

[7] Garcia RM, Kraay MJ, Goldberg VM. Isolated resurfacing of the previously unresurfaced patella total knee arthroplasty. J Arthroplasty 2010; 25(5): 754-8.

[8] Spencer SJ, Young D, Blyth MJ. Secondary resurfacing of the patella in total knee arthroplasty. Knee 2010; 17(3): 187-90. 
[9] Karnezis IA, Vossinakis IC, Rex C, et al. Secondary patellar resurfacing in total knee arthroplasty: results of multivariate analysis in two case-matched groups. J Arthroplasty 2003; 18(8): 993-8.

[10] Khatod M, Codsi M, Bierbaum B. Results of resurfacing a native patella in patients with a painful total knee arthroplasty. J Knee Surg 2004; 17(3): 151-5.

[11] Mockford BJ, Beverland DE. Secondary resurfacing of the patella in mobile-bearing total knee arthroplasty. J Arthroplasty 2005; 20(7): 898-902.

[12] Muoneke HE, Khan AM, Giannikas KA, et al. Secondary resurfacing of the patella for persistent anterior knee pain after primary knee arthroplasty. J Bone Joint Surg Br 2003; 85(5): 6758.
[13] Ahmad R, Kumar GS, Katar K, et al. Significance of a "hot patella" in total knee replacement without primary patellar resurfacing. Knee 2009; 16(5): 337-40.

[14] Munoz-Mahamud E, Popescu D, Nunez E, et al. Secondary patellar resurfacing in the treatment of patellofemoral pain after total knee arthroplasty. Knee Surg Sports Traumatol Arthrosc 2011; 19(9): 1467-72.

[15] Porteous A. The patella in primary and revision total knee arthroplasty. Orthop Trauma 2012; 26(2): 86-90.

[16] Daniilidis K, Vogt B, Gosheger G, et al. Patellar resurfacing as a second stage procedure for persistent anterior knee pain after primary total knee arthroplasty. Int Orthop 2012; 36(6): 1181-3.

[17] Maloney WJ. The stiff total knee arthroplasty: evaluation and management. J Arthroplasty 2002; 17(4 Suppl 1): 71-3.

[18] Scuderi GR. The stiff total knee arthroplasty: causality and solution. J Arthroplasty 2005; 20(4 Suppl 2): 23-6.

Received: June 26, 2012

Revised: July 25, 2012

Accepted: August 3, 2012

(C) Correia et al.; Licensee Bentham Open.

This is an open access article licensed under the terms of the Creative Commons Attribution Non-Commercial License (http://creativecommons.org/licenses/by-nc/3.0/) which permits unrestricted, non-commercial use, distribution and reproduction in any medium, provided the work is properly cited. 